\title{
Screening for Fetal Malformations
}

\author{
M Theodora, G Daskalakis, A Antsaklis
}

Fetal Medicine Unit 1st, Department of Obstetrics and Gynecology

University of Athens, Greece

Alexandra Hospital

\begin{abstract}
Even though the issue of routine sonography for low-risk pregnant women continues to be controversial mainly because randomized trials have failed to demonstrate clear benefit on pregnancy outcome, detection of different fetal malformations seems be one of the main indications for performing scans in pregnancy. Due to progress made in ultrasound technology and the experience of sonographers it is feasible to diagnose congenital anomalies as early as the end of the first trimester. Nevertheless most targeted studies are performed at 18 to 20 weeks of gestation. The highest rates of detection of congenital anomalies are seen in tertiary care settings such as a university medical center. In high-risk cases a consulting perinatologist is commonly the physician most likely to integrate the ultrasound findings.
\end{abstract}

Key words: Fetal malformation, screening, ultrasound.

\section{INTRODUCTION}

Obstetric ultrasonography has become an important part of routine antenatal care in many countries mainly in Europe. Fetal malformations are the cause of $30 \%$ of perinatal deaths in developed countries, even though their prevalence is relative low, ranging from 2 to $4 \%$ of all births. Ultrasound for fetal anomalies was introduces in obstetric in 1979. ${ }^{1}$ Although one of the most common indications of ultrasound examination during pregnancy is screening for fetal anomalies, the greatest risk of antenatal sonography is misdiagnosing of such an abnormality. A false positive diagnosis of a malformation may lead to parental anxiety but these errors are usually corrected during a second examination in a tertiary referral center. On the other hand a missed diagnosis (false negative) usually remains undetected until delivery, unless the patient undergoes an additional examination for another indication.

Antenatal sonography is well accepted by patients and their families and often pregnant women estimate the adequacy of their perinatal care by the frequency of ultrasound examinations they undergo. Additionally many couples like to have videotapes as "souvenirs" of their antenatal ultrasound studies.

When congenital anomaly is recognized at delivery one of the main concerns is: whether we could have seen this on ultrasound before delivery? It is therefore important to understand what the expectations should be for both the parents and the obstetricians. Indications for ultrasound in pregnancy are well known and guidelines have been established. Obstetrics sonography should be performed at an appropriate gestational age by an experienced practitioner. It is estimated that $70 \%$ of obstetrical patients in the USA undergo ultrasound fetal examination at least one time during pregnancy whereas in Greece the estimated percentage is even bigger than that.

Basic ultrasound examination is performed most often for the purpose of biometry and the establishment of gestational age. On the other hand more detailed ultrasound examinations are preformed during pregnancy in order to detect fetal anomalies in fetuses. Various descriptive terms have been used to describe such a detailed study including level II comprehensive, extended and targeted. These tests are preformed in women at high-risk as well as women at low-risk of having a malformed fetus.

Past medical history or factors identified in the index pregnancy are indications for a high-risk pregnancy. In these cases pregnant women do expect to have information about baby's health and in case a congenital anomaly is present they want to known the prognosis, the treatment and the recovery options. In these cases ultrasound examinations are justified and well accepted by the obstetricians since their effect on pregnancy outcome is positive.

On the contrary there is a divergence of opinion between American and European obstetricians on the necessity of universal ultrasonography in low-risk pregnancies. In most European countries, ultrasonography is mandated for every pregnancy without regard to risk status. For example, in Germany it is mandated than pregnant women should be offered an ultrasound scan for each trimester and there is a referral system organized in case a fetal anomaly is suspected. ${ }^{2}$ In general, European doctors have chosen promptly to scan all pregnant women on a routine basis, very quickly reaching high compliance rates. Several consensus conferences took place in Europe and all emphasizing the likely favorable rate of ultrasound examination of low-risk population. Routine use of ultrasound in low pregnancies has been offered for the decrease of labor inductions performed for postdatism, for the early detection of multifetal gestations, for detection of placental 
implantation abnormalities and for the antenatal diagnosis of congenital anomalies.

On the contrary the question of whether or not all pregnant women should have an ultrasound examination continuous to be controversial in the USA.

The RADIUS trial ${ }^{3}$ is a randomized trial comparing routinely scanned pregnant patients with those having ultrasound only when indicated. It represented one of the largest sample of population studied. This study remains the most expensive and one of the largest prospective studies of the routine versus indicated use of obstetrical ultrasound. Additionally is one of the most controversial study in obstetrical ultrasound and it has been used as an example of the poor performance of ultrasound in detecting fetal abnormalities. ${ }^{4}$ In this study which was preformed between 1987 and 1991, 15.151 low-risk pregnant women with no clear indication for an ultrasound examination were randomized to one of two groups. The ultrasound screening group underwent two planned ultrasound examinations: The first at 15 to 22 weeks and the second 31 to 35 weeks. The control group had an ultrasound examination if and when there was an indication for such test. In this control group $60 \%$ of the patients ended up having an ultrasound. The patients in the study group were examined at one of the 28 ultrasound laboratories participating in the study. Congenital anomalies were diagnosed antenatally more commonly in the ultrasound screening group (35\%) as opposed to the control group (11\%). Of the detected anomalies only $17 \%$ were detected prior to 24 weeks of gestation. The writers also separated out those abnormalities that were potentially detectable with ultrasound and using these criteria, $47 \%$ of anomalies were identified in total and $24 \%$ before 24 weeks of pregnancy. Finally, the study looked in the sensitivity of detection in tertiary centers before 24 weeks which was 35\% compared to $13 \%$ in nontertiary centers at the same gestational age. There was a difference in anomaly detection between the study group and the control group but this difference was not projected on the perinatal mortality and morbidity rate between the two groups. Thus according to the writers, the study has not shown a clear benefit of routine as opposed to indicated ultrasound studies in patients considered to be at low-risk. Additionally the financial cost of performing routine ultrasound is a prominent feature in the argument of those who believe that such studies should not be offered to low-risk women. It has been suggested that an additional USD 500 millions per year would be required to provide routine sonography for all pregnant women in the USA. The ACOG supported the conclusion of RADIUS study and the college stated that "in USA the routine use of ultrasound cannot be supported from a cost benefit stand point and that the college does not recommend routine ultrasound screening". It should be noted that a lot of criticisms have pointed out scientific shortcomings in both the methods and conclusions of the RADIUS trial mainly on the low rate of detection of congenital anomalies has been made.
Eurocat $^{5}$ and Eurofetus ${ }^{6}$ databases are used in Europe for registering and evaluating fetal anomalies. Eurocat is a register that records congenital anomalies. The Eurofetus trial was conducted between 1990 and 1993 in 61 European centers. It was the largest prospective screening study since it included 170,800 pregnant women. All women enrolled in the study had a scan between 18th and 22nd week of pregnancy. The mean number of ultrasounds for each woman was three and the overall sensitivity for detecting malformations was 56.2\%. Of 3685 malformed fetuses, 2262 had been diagnosed during pregnancy (sensitivity 61.4\%). The detection rate was higher for major malformations than for minor anomalies (73.7\% and $45.7 \%$ respectively). The detection rate for selected anomalies can be seen in Table 1. Fifty-five percent of the major anomalies were detected and diagnosed prior to the 24th week of pregnancy (Table 2).

The results of Eurofetus study are less impressive of some European Ultrasound Units. It has been shown that sensitivity significantly increases at successive intervals. On top of detecting structural anomalies, it has been estimated that antenatal examination could reveal three quarters of chromosomal abnormalities and nearly all cases of trisomies 13 and 18. Although maternal age is not included in the list of indications for ultrasonography, it seems that the maternal risk for trisomy 21 may be adjusted by the use of fetal ultrasonographic markers and it has been suggested a second trimester genetic sonogram for the adjustment of risk for trisomy 21.

Based on these data many pregnant women at or above the age of 35, either decline genetic amniocentesis or would prefer to have a detailed ultrasound examination prior to deciding whether to proceed with amniocentesis nowadays.

\section{Screening for Anomalies in the First-trimester}

The new treads in fetal anomalies screening include the firsttrimester sonographic scanning (11 to 14 weeks) for full fetal anatomy evaluation through transvaginal or transabdominal ultrasonography and early detection of many severe congenital malformations along with the evaluation of nuchal translucency thickness. Although the first-trimester screening could not completely replace the standard mid trimester scan for structural anomalies, different studies have shown that it possible to identify fetal abnormalities and detect genetic syndromes in the first-trimester scan (Fig. 1).

The feasibility of detection of fetal anomalies early in pregnancy was achieved due to the advances in ultrasound technology as well as the increasing knowledge and experience of sonographers. Early pregnancy ultrasound was initially introduced for determining fetal availability and accurate pregnancy dating. It has been also shown that measurement of fetal nuchal translucency (NT) thickness between 11 and 
M Theodora et al

Table 1: Detection sensitivity as function of malformations

\begin{tabular}{|c|c|c|c|}
\hline Anomaly & Number & True positive & Sensitivity \\
\hline Conjoined twins & 5 & 4 & - \\
\hline Multiple congenital anomalies & 115 & 86 & 74.8 \\
\hline \multicolumn{4}{|c|}{ CENTRAL NERVOUS SYSTEM ANOMALIES } \\
\hline Central nervous system anomalies & 738 & 652 & 88.3 \\
\hline \multicolumn{4}{|l|}{ HEART AND GREAT VESSEL ANOMALIES } \\
\hline Total major anomalies of heart & 366 & 142 & 38.8 \\
\hline Total minor anomalies of heart & 587 & 122 & 20.8 \\
\hline Total heart and great vessel anomalies & 953 & 264 & 27.7 \\
\hline \multicolumn{4}{|l|}{ DIGESTIVE SYSTEM ANOMALIES } \\
\hline Occlusion & 8 & 8 & - \\
\hline Total minor anomalies of digestive system & 221 & 115 & 52.0 \\
\hline Total digestive system anomalies & 229 & 123 & 53.7 \\
\hline \multicolumn{4}{|l|}{ URINARY TRACT ANOMALIES } \\
\hline Total major anomalies of urinary tract & 138 & 117 & 84.8 \\
\hline Total minor anomalies of urinary tract & 816 & 844 & 89.1 \\
\hline Total urinary tract anomalies & 954 & 844 & 88.5 \\
\hline \multicolumn{4}{|l|}{ MUSCULOSKELETAL ANOMALIES } \\
\hline Total major musculoskeletal anomalies & 349 & 257 & 73.6 \\
\hline Total minor musculoskeletal anomalies & 694 & 125 & 18.0 \\
\hline Total musculoskeletal anomalies & 1034 & 382 & 36.6 \\
\hline \multicolumn{4}{|l|}{ MISCELLANEOUS } \\
\hline Total cleft lips and palates & 316 & 57 & 18 \\
\hline \multicolumn{4}{|l|}{ RESPIRATORY SYSTEM ANOMALIES } \\
\hline Total major respiratory system anomalies & 14 & 11 & - \\
\hline Total minor respiratory system anomalies & 16 & 10 & \\
\hline Total respiratory system anomalies & 30 & 21 & 70 \\
\hline Total other anomalies of head and neck & 42 & 6 & 14.3 \\
\hline Total congenital anomalies of ear and eye & 26 & 4 & 15.4 \\
\hline Total miscellaneous benign neoplasms & 1216 & 112 & 88.9 \\
\hline Ovarian cyst & 38 & 39 & 100 \\
\hline TOTAL ALL ANOMALIES & 4615 & 2593 & 56.2 \\
\hline
\end{tabular}

From Grandjean H, Larroque D, Levi S, and the Eurofetus Study Group. The performance of routine ultrasonographic screening of pregnancies in the Eurofetus Study. Am J Obstet Gynecol 1999;181:446-54. 
Table 2: Average gestational age at diagnosis for isolated malformations

\begin{tabular}{|c|c|c|c|c|}
\hline Anomaly & No. & Gestational age (wk) & $S D$ & Before $24 w k^{*}(\%)$ \\
\hline \multicolumn{5}{|c|}{ CENTRAL NERVOUS SYSTEM ANOMALIES } \\
\hline Anencephaly & 128 & 19.1 & 4.5 & 82.8 \\
\hline Encephalocele & 21 & 22.8 & 7.5 & 66.7 \\
\hline Hydrocephalus & 110 & 27.9 & 7.2 & 35.5 \\
\hline Other CNS anomalies & 144 & 25.7 & 7.2 & 42.3 \\
\hline \multicolumn{5}{|c|}{ HEART AND GREAT VESSEL ANOMALIES } \\
\hline Major anomalies & 76 & 26.8 & 7 & 40.7 \\
\hline Minor anomalies & 48 & 30.7 & 5.5 & 5.1 \\
\hline \multicolumn{5}{|c|}{ DIGESTIVE SYSTEM ANOMALIES } \\
\hline Digestive system anomalies & 85 & 30.2 & 5.8 & 12.5 \\
\hline \multicolumn{5}{|c|}{ URINARY TRACT ANOMALIES } \\
\hline Major anomalies & 66 & 23 & 6.5 & 56.8 \\
\hline Minor anomalies & 582 & 29.4 & 6.5 & 27.7 \\
\hline \multicolumn{5}{|c|}{ MUSCULOSKELETAL ANOMALIES } \\
\hline Major anomalies & 173 & 23.4 & 7.2 & 62.9 \\
\hline Minor anomalies & 48 & 23.4 & 5.3 & 59.6 \\
\hline \multicolumn{5}{|c|}{ MISCELLANEOUS } \\
\hline Cleft palates and cleft lips & 40 & 28 & 7.4 & 31.6 \\
\hline Hemangioma any site & 78 & 17 & 4.8 & 92.3 \\
\hline Ovarian cyst & 37 & 34.2 & 3.2 & 0 \\
\hline \multicolumn{5}{|c|}{ TOTAL } \\
\hline TOTAL ISOLATED ANOMALIES & 1675 & 26.4 & 7.4 & 41.1 \\
\hline
\end{tabular}

Details concern malformations for which average age at diagnosis differs from whole category

*Rate of diagnosis before 24 weeks.

From Grandjean H, Larroque D, Levi S, and the Eurofetus Study Group. The performance of routine ultrasonographic screening of pregnancies in the Eurofetus Study. Am J Obstet Gynecol 1999;181:446-54.

14 weeks' gestation can identify $75 \%$ of fetuses affected with trisomy 21 and other chromosome abnormalities in unselected population ${ }^{7}$ and that increased nuchal translucency is associated with structural defects. ${ }^{8,9}$ Since the $11^{+0}$ to $13^{+6}$ weeks' scan has been established as a screening test for chromosomal abnormalities, a number of studies for assessment fetal anatomy (Fig. 1) and for screening for structural defects (Figs 2 and 3) at this gestational age have been published.

McAuliffe et a ${ }^{10}$ examined 300 singlenton fetuses between $1^{+0}$ and $13^{+6}$ weeks of gestation. In their study a complete anatomy survey was successful in 33\% of these fetuses. The structural visibility rates are seen in Table 3 . Of a total of 6 congenital defects in this study only one (a neural tube defect) was detected in the first trimester, 4 at the 18 to 20 week anatomic scan, and 1 postnatally. The authors concluded that since some anomalies are not evident in the first trimester, the 18 to 20 week scan remains the gold standard.
Souka et a ${ }^{11}$ examined 1148 low-risk pregnancies in order to assess the feasibility of examining cardiac and noncardiac fetal anatomy in the setting of the routine 11 to 14 week ultrasound scan. In this study complete examination of the fetal anatomy was achieved in $48 \%$ of the fetuses, whereas noncardiac anatomy was examined successfully in $86 \%$ of the fetuses (Table 4). Souka et $\mathrm{al}^{12}$ also estimated the sensitivity of the first and second trimester ultrasound scan in the detection of major anomalies. In this study a total of 1148 singleton pregnancies were examined. Seven of the 14 major anomalies were detected at the 11 to 14 weeks scan, and an additional 6 at the second trimester scan. The sensitivity of the early anatomic examination was $50 \%$ and the overall of the two stage study was $92.8 \%$

All studies agree that the detection rates of fetal abnormalities in first-trimester increase as the CRL of the fetuses increase, when the nuchal translucency is above the 95th 
Table 3: Standardized protocol and structural visibility rates for first-trimester fetal anatomy

\begin{tabular}{|c|c|c|c|}
\hline Structure & Protocol for satisfactory visualization & \# Seen & $\%$ \\
\hline Cranium & Presence of ossified cranial bones & $324 *$ & 99.7 \\
\hline Intracranial anatomy & Midline present and choroid plexi seen in ventricles & 319 & 98 \\
\hline Face & Presence of nasal bones, orbits and a normal profile & 308 & 95 \\
\hline Heart and ventricles & $\begin{array}{l}\text { Heart in left side of thorax and apex pointing to left, } \\
4 \text { chambers identified with symmetric atria }\end{array}$ & 274 & 84 \\
\hline Stomach & Stomach seen as an echo-free area in left upper quadrant & 319 & 98 \\
\hline Cord insertion & Normal cord insertion and normal abdominal wall & 322 & 99 \\
\hline Bladder & Bladder seen as an echofree area in pelvis & 291 & 89.5 \\
\hline Spine/back & $\begin{array}{l}\text { Intact skin overlying back. Spine intact from neck to } \\
\text { sacrum in both transverse and longitudinal planes }\end{array}$ & 147 & 45 \\
\hline Lower limbs & All long bones visible, correct posture of feet & 323 & 99 \\
\hline Upper limbs & All long bones visible, correct posture of hands & 323 & 99 \\
\hline Complete anatomy seen & & 109 & 33 \\
\hline
\end{tabular}

* The fetus where cranial ossification was not seen had a CRL of $46 \mathrm{~mm}$; cranium was seen at 18 weeks ultrasound.

From McAuliffe FM, Fong KW, Toi A, Chitayat D, Keating S, Johnson JA. Ultrasound detection of fetal anomalies in conjunction with first-trimester nuchal translucency screening: A feasibility study. American Journal of Obstetrics and Gynecology 2005;193,1260-65.

percentile and when the scan is performed by an experienced sonographer.

\section{Ultrasound Scans as a Screening Test for Detection of Fetal Malformations}

The indications for an ultrasound scan are related to the time that the scan is performed. In cases where precise estimation of gestational age is required, ultrasound scan should be performed in the first-trimester or as early in pregnancy as feasible. The high frequency transvaginal probes, has greatly improved the diagnostic potential of ultrasound during the first-trimester and the early detection of congenital anomalies. Eighteen to twenty weeks is the traditional and appropriate time to perform a comprehensive anatomy scan (Figs 4 to 6). This ultrasound study allows a detailed review of fetal anatomy and is early enough so that amniocentesis or other diagnostic procedures can be performed prior to fetal viability. The genetic sonogram is a targeted study with special emphasis on ultrasonographic markers that may indicate aneuploidy. Targeted ultrasonography at 18 to 20 weeks allows the couple to consider all of their options and allows for appropriate referral and counseling.
However, some malformations are not easily visualized at this period. Hydrocephalus, bowel atriasias may develop after this period and may not be demonstrable until after 24 week's gestation. The optimal time for fetal echocardiography is probably the 20 to 22 weeks' gestation. Sonography may be required in the third trimester as well. This examination is ordered for cases with suspected intrauterine growth restriction, vaginal bleeding or fetal macrosomia and in some cases fetal malformations may be detected during this trimester as well.

Management of a malformed fetus is determined by different parameters. After the anomaly is diagnosed additional tests are required before any decisions such as termination or intrauterine therapy are planned. Most of the times the above mentioned process provokes a lot of anxiety to the parents especially in cases where the anomaly detected is not a clear indication for termination of the pregnancy or it is not a strong marker for a disease. Not screening low-risk pregnancies, while avoiding this anxiety will deny parents their right to know if their child carries an anomaly. From the ethical point of view, screening respects the autonomy of the couple in deciding if they are going to continue or terminate a pregnancy with a malformed fetus. 


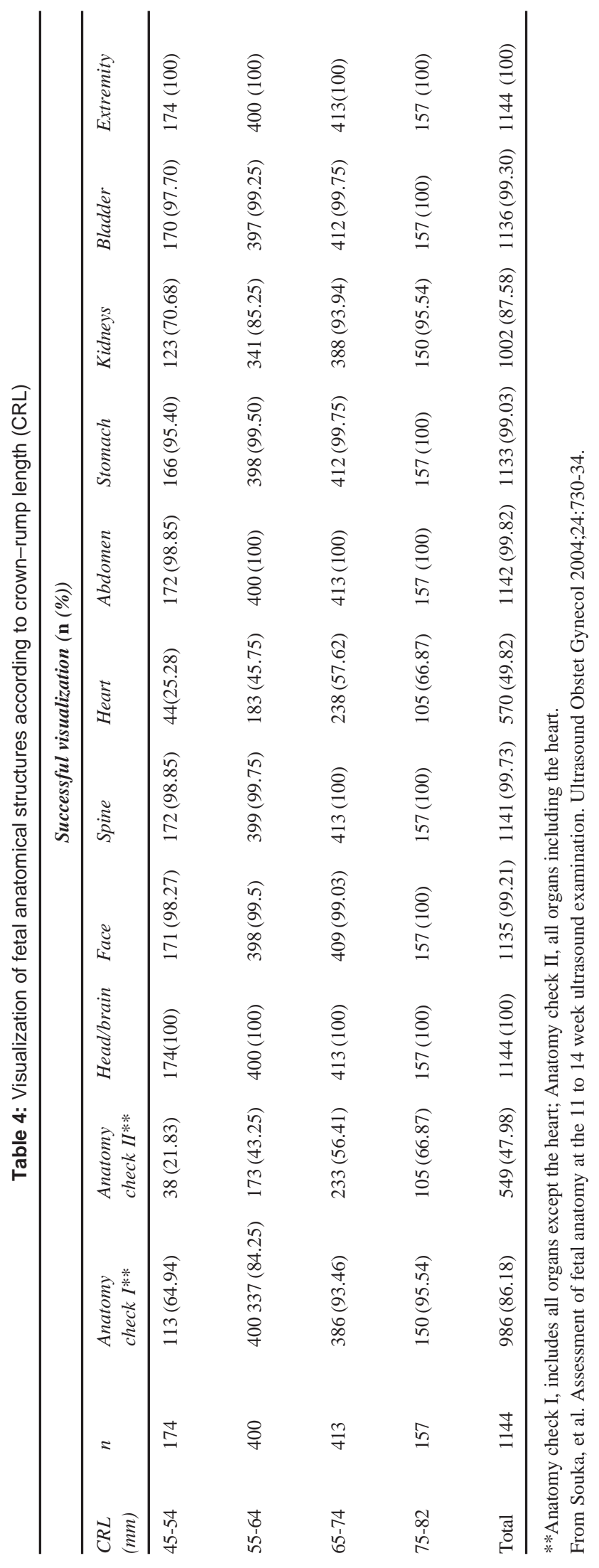


M Theodora et al
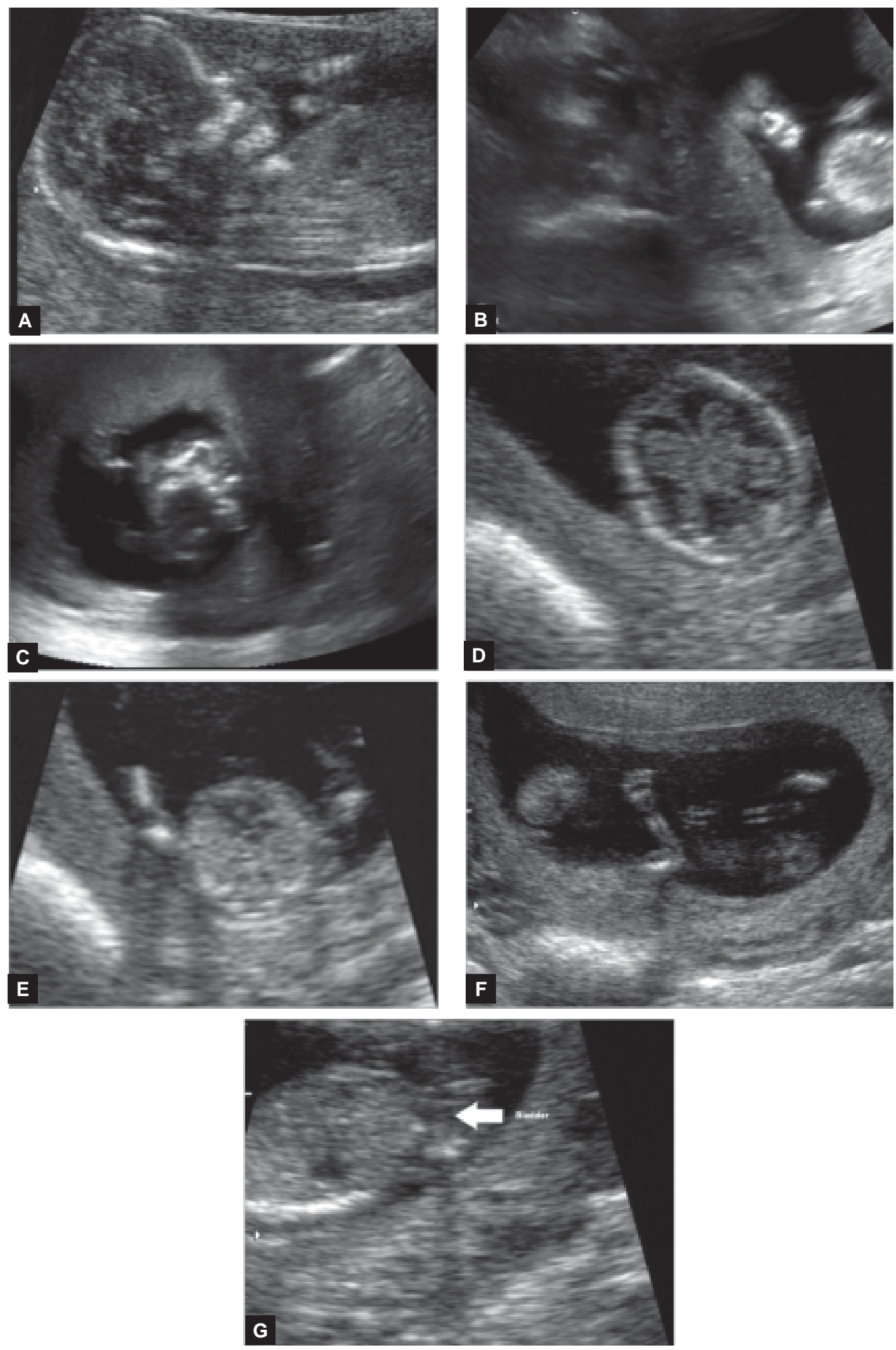

Figs 1A to G: Normal anatomy in first-trimester 

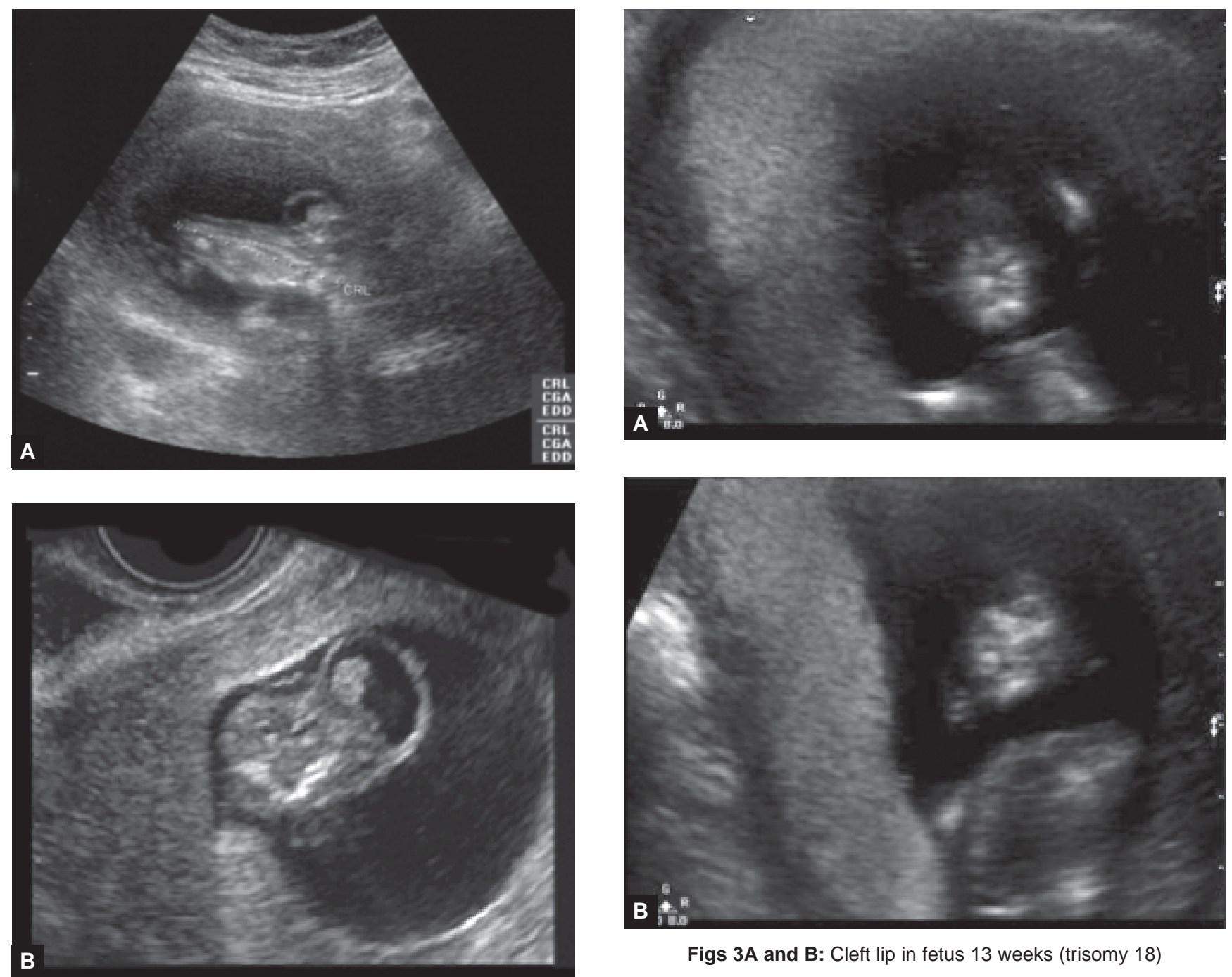

Figs 3A and B: Cleft lip in fetus 13 weeks (trisomy 18)

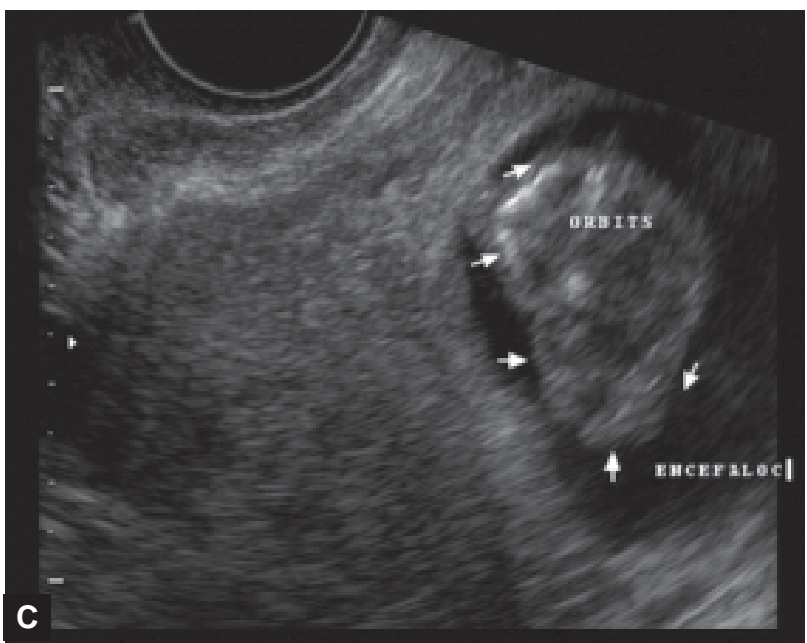

Figs 2A to C: Enchephalocele in fetus $11^{+5}$ weeks

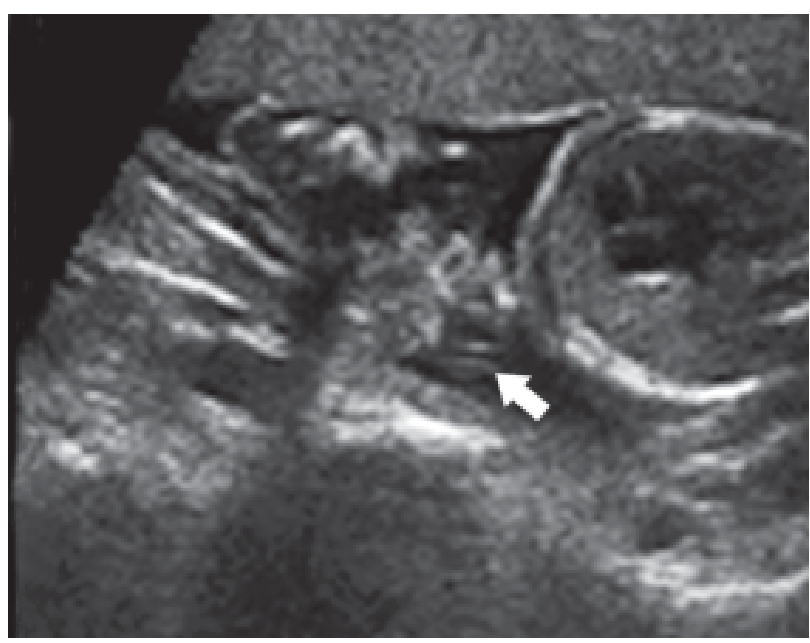

Fig. 4: Gastroschisis in 22 weeks.

Free intestine inside amniotic cavity (arrow) 


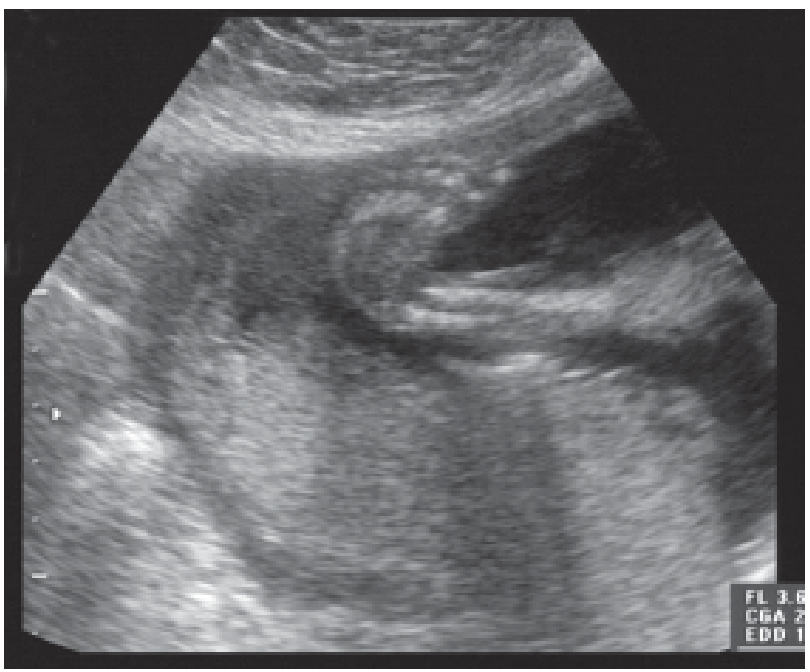

Fig. 5: Clubfoot in 22 weeks

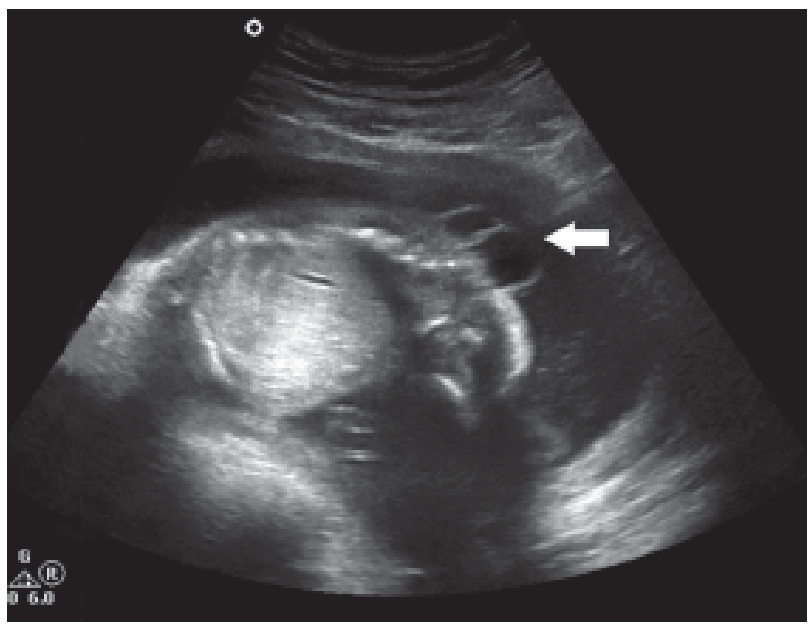

Fig. 6: Spina bifida (arrow) 20 weeks

Additionally many fetuses maybe saved if timely treatment in utero or after delivery is given. Seventy percent of fetuses with cardiac arrhythmias will survive after birth if transplacental treatment is given. ${ }^{13}$ Also pyelectasis before birth can identify fetuses at risk for progressive deterioration of renal function within the first months of life, requiring neonatal surgery. ${ }^{14}$

\section{Limitations of Ultrasound as Screening Test}

Although it is generally accepted that experience is needed in order to achieve high detection rates when performing ultrasound scans during pregnancy, antenatal sonography is performed in different medical centers, doctor's offices, hospitals and by physicians of varying levels of experience or by technicians. In USA and Greece it is not unusual for obstetricians-gynecologists to have ultrasound equipment in their private offices. Radiologists usually have either a hospital based practice or function in a free standing diagnostic imaging center. The experience of the obstetrician clinician with sonography must begin with detailed knowledge regarding fetal cross-sectional anatomy. It is important for the clinician to know his or her limits with regard to the use of ultrasound. Some clinical situations are encountered very rarely in the community practice of obstetrics but are commonly seen in a tertiary referral center. Basic and limited scans preformed in an obstetrician's office could provide useful first-stage information and a fetus with a structural malformation could be then referred for confirmation of the defect as well as for a detailed search for other malformations to a tertiary center. Unfortunately it has been reported that of the first-trimester studies carried out in obstetric practice, only $35 \%$ met all the ACOG criteria ${ }^{15}$ and only $15 \%$ all the AIUM $^{16}$ criteria for documentation. Firsttrimester reports from radiology practices met ACOG criteria in $11.5 \%$ of cases and AIUM criteria in $4 \%$ of cases. Incredibly only $52 \%$ of these second and third trimesters studies documented the four chamber view of the heart and only $15 \%$ documented an intact anterior abdominal wall. There is clear evidence from the RADIUS study that the experience of the examiner is an important determinant in the ultrasound diagnosis of congenital malformations. It seems safe to conclude that if the primary indication for obstetric sonography is to diagnose or exclude congenital anomalies, the study should be performed in a center where this special expertise has been demonstrated. It has been proved that ultrasound scan is not effective for detecting fetal malformation if used at a basic level since trained personnel is need to indentily anomalies more frequently. ${ }^{3}$ There is good evidence to support the recommendation that the sensitivity of the ultrasound screening in detecting fetal malformations in low-risk pregnancies cannot be established with precision. Therefor its use will continue to be decided on a local level and varies in different centers with different level of operators training and financial resources.

Limitations of obstetrical ultrasonography should be briefly reviewed with patients prior to the initiation of the procedure. It should be noted that some major malformations are easily detectable whereas other malformations present subtle ultrasound images or may not be diagnosable in the time of the scan, e.g. midtrimester. In a study performed in United States tertiary referral center it has been reported the sensitivity of ultrasound in the detection of structural defects to be $53 \%$ over all and $89 \%$ for the common lethal malformations. The sensitivity increased with gestational age (47\% below 20 weeks, $59 \%$ at 20 weeks and $68 \%$ at greater than 24 weeks) and with the indication for sonography (routine examination having the lowest sensitivity at 36\%). Fetal position, oligohydramnios and obesity may obscure proper visualization of fetal structures and may make a definitive diagnosis impossible. In cases with increased risk of fetal congenital anomalies, it should be stress out that diagnose is made most consistently in tertiary care 
ultrasound facilities and not in obstetrics offices or in radiology departments.

Targeted sonography should be performed only by those physicians with training and experience in the diagnosis of congenital anomalies. This examination requires a detailed base of knowledge regarding the fetal effects that may ensue from particular maternal medical illnesses or from familial genetic conditions. Ultrasound is used not only for diagnosis but as a tool for the management of a complicated pregnancy and for this reason the perinatologist is perfectly the right doctor to provide sonographic diagnosis and plan the management of a high-risk pregnancy.

\section{CONCLUSION}

The issue of routine sonography for low-risk pregnant women continues to be controversial since randomized trials have not been able to demonstrate a clear benefit. Ultrasound seems not to meet all the requirements for screening program since experience is need in order to achieve acceptable high rates in fetal malformation diagnosis. Nevertheless routine scan preformed by specialized doctors in tertiary care settings seems to be justified because of the advantages and the information which are provided by the early diagnosis of fetal abnormalities. In this way timely management of the affected pregnancy is possible. Although great progress is being made in the firsttrimester diagnoses of congenital anomalies, most targeted studies are performed at 18 to 20 weeks of gestation. In highrisk cases a consulting perinatologist is commonly the physician most likely to integrate the ultrasound findings.

\section{REFERENCES}

1. Hobbins JC, Grannum PAT, Berkowitz RL, Silverman R, Mahoney MJ. Ultrasound in the diagnosis of congenital anomalies, 1979.

2. Salvator Levi. Ultrasound in prenatal diagnosis: Polemics around routine ultrasound screening for second trimester fetal malformations. Prenat Diagn 2002;22:285-95.

3. Crane JP, LeFevre ML, Winborn RC, et al. RADIUS Study group. A randomized trial of prenatal ultrasonographic screening: impact on the detection, management, and outcome of anomalous fetus. Am J Obstet Gynecol 1994;171:392-99.
4. Ewigman BG, Crane JP, Frigoletto FD, LeFevre ML, Bain RP, McNellis D. Effect of prenatal ultrasound screening in perinatal outcome. N Engl J Med 1993;329:821-27.

5. EUROCAT Working Group. Surveillance of congenital anomalies in Europe 1980-1999: Report 8. EUROCAT central registry, University of Ulster, Northern Ireland BT370QB. 2003.

6. Grandjean H, Larroque D, Levi S, and the Eurofetus team. The performance of routine ultrasonographic screening of pregnancies in the Eurofetus study. Am J Obstet Gynecol 1999;181:446-54.

7. Snijders RJM, Noble P, Sebire N, Souka A, Nicolaides KH. Multicentre project on assessment of risk of trisomy 21 by maternal age and fetal nuchal-translucency thickness at 10-14 weeks of gestation. Lancet 1998;352:343-46.

8. Souka AP, Snijders RJM, Novakov A, Soares W, Nicolaides $\mathrm{KH}$. Defects and syndromes in chromosomally normal fetuses with increased nuchal translucency thickness at 10-14 weeks gestation. Ultrasound Obstet Gynecol 1998;11:391-400.

9. McAuliffe F, Hornberger L, Winsor S, Chitayat D, Chong K, Johnson J. Fetal cardiac defects and increased nuchal translucency thickness. Am J Obstet Gynecol 2004;191:148690.

10. McAuliffe FM, Fong KW, Toi A, Chitayat D, Keating S, Johnson JA. Ultrasound detection of fetal anomalies in conjunction with first-trimester nuchal translucency screening: A feasibility study. American Journal of Obstetrics and Gynecology 2005;193,126065.

11. Souka AP, Pillalis A, Kavalakis Y, Kosmas Y, Antsaklis P, Antsaklis A. Assessment of fetal anatomy at the 11 to 14 week ultrasound examination. Ultrasound Obstet Gynecol 2004;24:730-34.

12. Souka AP, Pilalis A, Kavalakis I, Antsaklis P, Papantoniou N, Mesogitis S, Antsaklis A. Screening for major structural abnormalities at the 11 to 14 week ultrasound scan. Am J Obstet Gyn 2006;194:393-96.

13. Hajdu J, Marton T, Toth PE. Congenital heart abnormalities and cardiac dysfunction: How prenatal diagnosis changed the chances for survival. Orv Hetil 140:815-18.

14. Gotoh H, Masuzaki H, Fukuda H, Yoshimura S, Ishimaru T. Detection and assessment of pyelectasis in the fetus: Relationship to postnatal renal function. Obstet Gynecol 1998;92:226-31.

15. ACOG practice patterns. Routine ultrasound in low-risk pregnancies. Int J Gynecol Obstet 1997;59:273-78.

16. Laurel MD. American Institute of Ultrasound in Medicine. Guidelines for the performance of the antepartum obstetrical examination, 1994. 